\title{
Terahertz and photonics seamless networks
}

\author{
Tetsuya Kawanishi ${ }^{1,2}$ \\ ${ }^{1}$ Waseda University, Shinjuku, Tokyo, Japan \\ ${ }^{2}$ National Institute of Information and Communications Technology, Koganei, Tokyo, Japan \\ e-mail: kawanishi@waseda.jp
}

\begin{abstract}
Radio links using terahertz (THz) waves can provide high-speed wireless transmission whose bitrate is higher than $100 \mathrm{~Gb} / \mathrm{s}$. Congestion of radio spectrum in $\mathrm{THz}$ bands $(0.1-$ $10 \mathrm{THz}$ ) is not so significant for the time being. However, multilevel modulation formats would be required in in $\mathrm{THz}$ bands as well as in conventional millimeter-wave or microwave bands, to increase spectral efficiency. We provide overviews on spectral efficiency and transmission capacity of high-speed radio links using THz bands. Reduction power consumption in radio equipment is also very important to reduce operation cost of networks. We describe a survey result on power consumption of short-distance wireless systems. The result implies that $\mathbf{T H z}$ high-speed radio links would be useful to reduce power consumption per bit in transmission. However, transmission distances in THz systems would be shorter than a few kilometers due to attenuation in the air. Thus, we should rely on seamless networks consisting of $\mathrm{THz}$ radio-links and optical fibers, where devices developed for optical fiber transmission can be used to THz wave generation and detection.
\end{abstract}

Keywords - THz, millimeter-wave, optical fiber, modulation, transmission

\section{INTRODUCTION}

$5 \mathrm{G}$ realized over $10 \mathrm{~Gb} / \mathrm{s}$ high-speed wireless data services, by using the "high-band," whose frequency is above $6 \mathrm{GHz}$, while the "low-band" is for macro or small cells on coexisting with conventional mobile networks [1]. Congestion of spectrum is significant at frequency bands lower than 100 $\mathrm{GHz}$ where it would be rather difficult to increase transmission capacity. In $\mathrm{THz}$ bands $(0.1-10 \mathrm{THz})$, wide range of spectrum is available for high-speed wireless transmission whose bitrate is over $100 \mathrm{~Gb} / \mathrm{s}$ [2, 3]. Due to large $\mathrm{THz}$ wave propagation loss, transmission distance would be shorter than a few hundred meters, so that a number of base stations (BSs) and remote antenna units (RAUs) are required to provide wide coverage [3].

Optical fiber transmission is commonly used to connect the BSs and RAUs. Hybrid networks consisting of optical fiber and radio-wave links would play important roles in future mobile networks where a number of BSs and RAUs should be connected. The radio-wave links use THz waves, to reduce the bitrate difference between optical fiber links and radiowave links. Thus, the hybrid networks would have many interfaces between photonic and THz links. Reduction of the cost and power consumption of the interfaces would be very important issue to realize the future mobile networks with low deployment and operation cost. In addition, latency reduction in the interference is also indispensable for lowlatency transmission which is required in mission-critical applications.

To reduce the latency and power consumption, the configuration of the interfaces should be as simple as possible $[3,4]$. Radio-over-fiber (RoF) which transfers waveforms for radio services over fibers can offer bridge between $\mathrm{THz}-$ waves in the air and optical signals in the fibers.

This paper provides overviews on spectral efficiency and transmission capacity of state-of-art $\mathrm{THz}$ systems. We define an index to measure impact on congestion mitigation, by a product of carrier frequency and spectral efficiency. The index shows that $300 \mathrm{GHz}$ radio links can provide over $100 \mathrm{~Gb} / \mathrm{s}$ high-speed transmission without losing spectral efficiency. This paper also describes a survey result on power consumption of short-distance wireless systems, to discuss that of $\mathrm{THz}$ transmission systems. Configurations of seamless networks are provided to discuss solutions for high-speed $\mathrm{THz}$ communications.

\section{CARRIER FREQUENCY AND SPECTRAL EFFICIENCY}

The use of $\mathrm{THz}$ carrier reduces spectral congestion in the frequency lower than $100 \mathrm{GHz}$. Enhancement of spectral efficiency relaxes the congestion as well. Occupancy of the $\mathrm{THz}$ region is so high as of now. However, the congestion in $\mathrm{THz}$ will be severe when the $\mathrm{THz}$ region is commonly used for various applications. We define a figure of merit by a product of carrier frequency and spectral efficiency, (CFSE: carrier frequency spectral efficiency product), to measure contribution to the congestion mitigation [3].

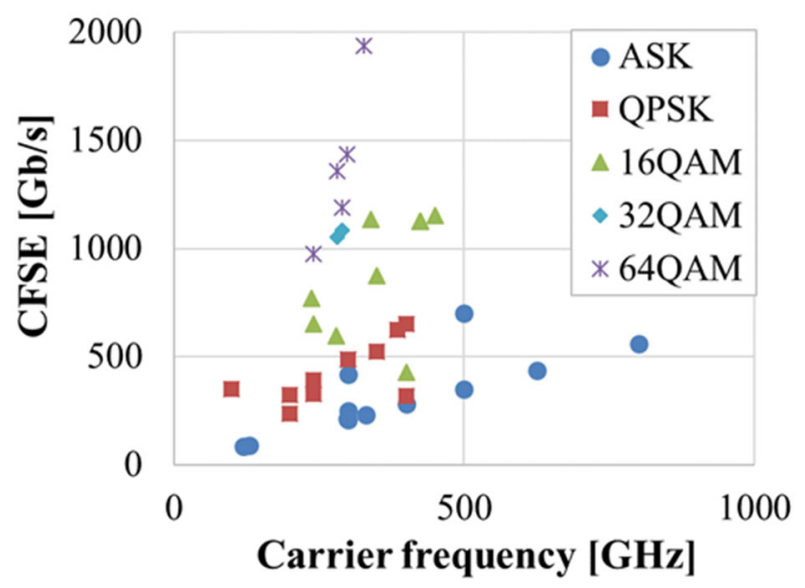

Figure 1 Carrier frequency spectral efficiency product (CFSE) as a function of carrier frequency [3]. 
Figs. 1 and 2 show the CFSE of the state-of-the-art THz transmission systems, where multi-level modulation formats such as quadrature phase shift keying (QPSK), 16 and 64 quadrature amplitude modulation (QAM) realize large CSFE [5-33]. The CFSE is proportional to the carrier frequency, when the spectral efficiency is constant. However, there is a peak at $300 \mathrm{GHz}$, as shown in Fig. 1. It shows that multi-level modulation technologies are not matured enough in frequency range over $300 \mathrm{GHz}$, where precise control of the $\mathrm{THz}$ waves is rather difficult as for now. The CFSE is useful to describe the status and trends of the high-speed transmission technologies. Fig. 2 shows the CFSE as a function of the data rate of the $\mathrm{THz}$ transmission systems. QPSK provides the largest data rate, while QAM realize large CFSE. High baud-rate QAM would be rather difficult in $\mathrm{THz}$ region.

From Figs. 1 and 2, we can conclude that the state-of-theart $\mathrm{THz}$ radio links can provide $100 \mathrm{~Gb} / \mathrm{s}$ high-speed transmission by the use of $300 \mathrm{GHz}$ frequency region, without losing the spectral efficiency.

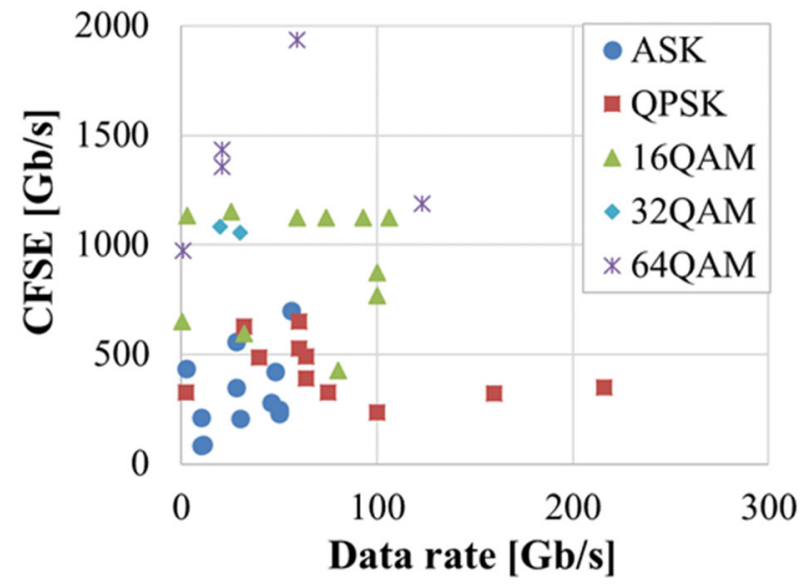

Figure 2 Carrier frequency spectral efficiency product (CFSE) as a function of data rate [3].

\section{POWER CONSUMPTION OF RADIO TRANSMITTERS}

Future mobile systems would require a number of BSs and RAUs, so that the power consumption reduction of the radio transmitters is very important to reduce the operation cost. Fig. 3 shows the power consumption per bit in various short distance radio transmission systems [3]. The power consumption is described as a function of the bitrate, where the horizontal axis is bitrate of a radio systems.

This plot is based on the specifications of the following wireless modules: Zigbee (NEC ZB24TM-Z2701), Bluetooth (Mitsumi WML-C75), IEEE 802.11n (Silex Technology SXSDMGN), IEEE 802.11ac (Silex Technology SX-SDMAC2832S+). For IEEE 802.11 ad and WirelessHD, we used specifications in Refs. [34, 35]. The power consumption of an optical transmitter (Finisar FTLC9152RGPL 100G 100m QSFP28 SWDM4 optical transceiver) is also plotted as a reference.

The total radio transmitter power consumption includes the power consumption at the power source, baseband unit for signal processing, radio front-end unit for radio-wave generation and detection, etc. That of particular functions, such as power supply, system management, packet buffering, should be proportional to time duration for transmission. The power consumption per bit would be inversely proportional to the bitrate, if such functions are dominant in the power consumption. Fig. 3 provides a curve fitting result given by

$$
\mathrm{y}=1.3 \times 10^{2} \times x^{-0.7}
$$

where the units of $x$ and $y$ axes are $n J$ and $\mathrm{Mb} / \mathrm{s}$, respectively. The curve fitting result has a difference from inverse proportion in the power index of $x^{0.3}$. The difference would be due to large power consumption in the rf front-end units in the transceiver modules.

Although the power consumption of electric circuits would be large in high-frequency operation, high-speed millimeterwave transmission systems such as 802.11ad and WirelessHD can provide low power consumption and highspeed wireless data links. As for now, data of the power consumption of high-speed radio transmission systems using $\mathrm{THz}$ are not available, but we may expect low-power consumption with high-bitrate transmission. Assuming that the power consumption of the $\mathrm{THz}$ system whose bitrate is $100 \mathrm{~Gb} / \mathrm{s}$ would follow the curve fitting result, the power consumption would be lower than conventional radio links and could be close to that of optical transmission.

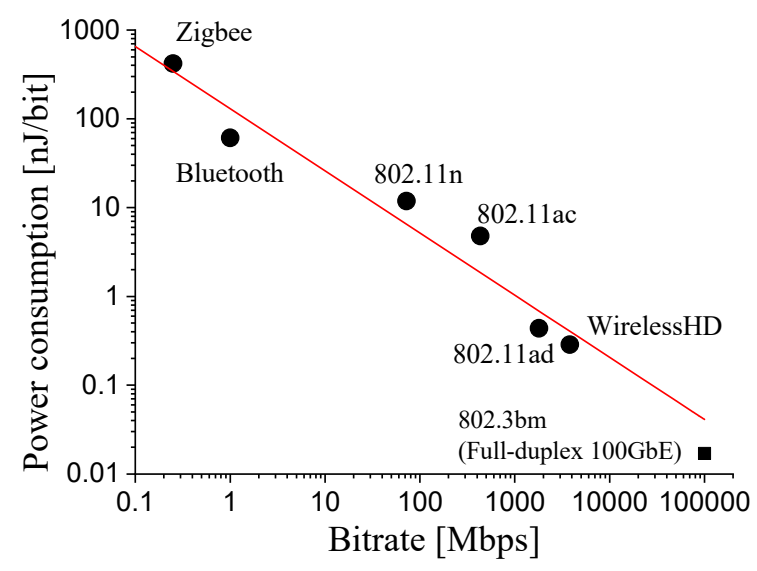

Figure 3 Radio transmitter power consumption [3].

\section{SEAMLESS NETWORKS}

Interfaces between photonic and $\mathrm{THz}$ links bridge high-speed optical and electric signals, by the use of optical-to-electric $(\mathrm{O} / \mathrm{E})$ and electric-to-optical (E/O) conversion. Fig. 4 shows a configuration of $\mathrm{THz}$ and photonics interfaces, where multilevel modulation formats such as QAM are utilized both in the optical fiber and radio links. Digital coherent technique is commonly used for multi-level signal detection, where the phase of the detected signal is estimated by using digital signal processing (DSP) units. As shown in Fig. 4, we need many DSP units both in the photonics and THz parts. Latency and power consumption in the DSP units would be an issue for mission-critical applications, while latency in $\mathrm{O} / \mathrm{E}$ and $\mathrm{E} / \mathrm{O}$ conversion is only from signal propagation delay in the conversion devices, so that it would be negligible smaller 
than that in DSP. On the other hand, RoF provides lowlatency conversion between radio-wave and optical signals, where waveforms for radio services transferred over fibers. In analog RoF, optical signals modulated by the waveforms are directly converted into radio-waves by using photodetectors. Thus, the total latency of optical and radio links including media conversion would be much smaller than in conventional systems with many DSP units. Even in RoF-based systems, DSP would be required for detection of multi-level signals or compensation of signal deformation [36]. However, the number of required DSP units would be minimized as shown in Fig. 5. Waveforms pass through the interfaces directly, while the DSP unit at the receiver provides comprehensive waveform compensation. At the RoF transmitter, a photonic local oscillator generates optical spectral component whose frequency separation equals the $\mathrm{THz}$ carrier frequency.

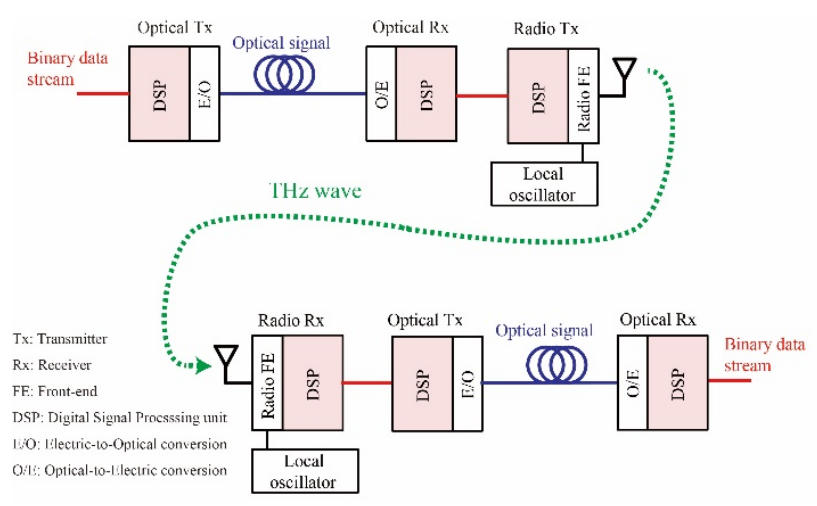

Figure 4 Configuration of $\mathrm{THz}$ and photonics interfaces, where coherent transmission is used both in radio and optical links.

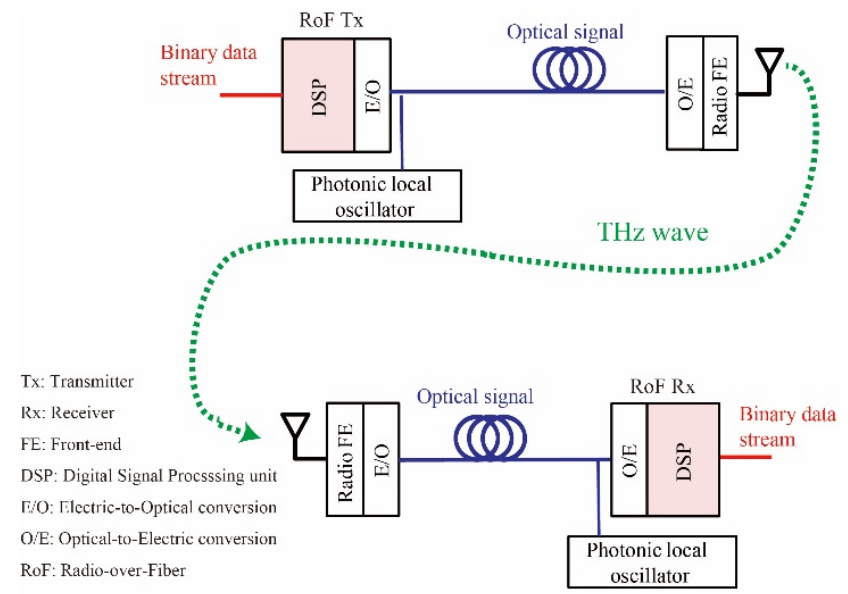

Figure 5 Configuration of $\mathrm{THz}$ and photonics interfaces, with RoF.

In RoF based systems as shown in Fig. 5, E/O and $\mathrm{O} / \mathrm{E}$ conversion devices should respond to the carrier frequency. However, it would be rather difficult to have direct $\mathrm{E} / \mathrm{O}$ or $\mathrm{O} / \mathrm{E}$ conversion in $\mathrm{THz}$ region. Fig. 6 shows a configuration of IF-over-fiber (IFoF) where the THz carrier is generated in the radio front-end unit by using a frequency multiplier [36]. The IFoF receiver generates local oscillator signals.

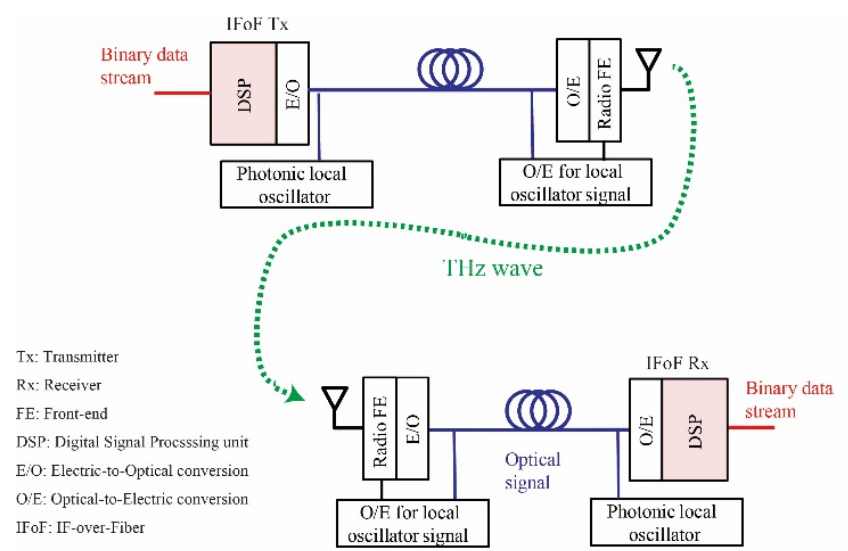

Figure 6 Configuration of $\mathrm{THz}$ and photonics interfaces, with IFoF.

Stability of the carrier frequency has a significant impact on radio transmission performance. $\mathrm{A} \mathrm{THz}$ carrier can be generated by two laser sources whose frequency separation is in $\mathrm{THz}$ region, however, the stability depends on that of the two lasers. The linewidth of the THz signal depends on that of the lasers. For stable operation, we need precise temperature and current control for lasers. If we use standard laser diodes designed for optical fiber communications, the linewidth would be a few MHz. The frequency excursion would be much larger than in typical electronic oscillators.

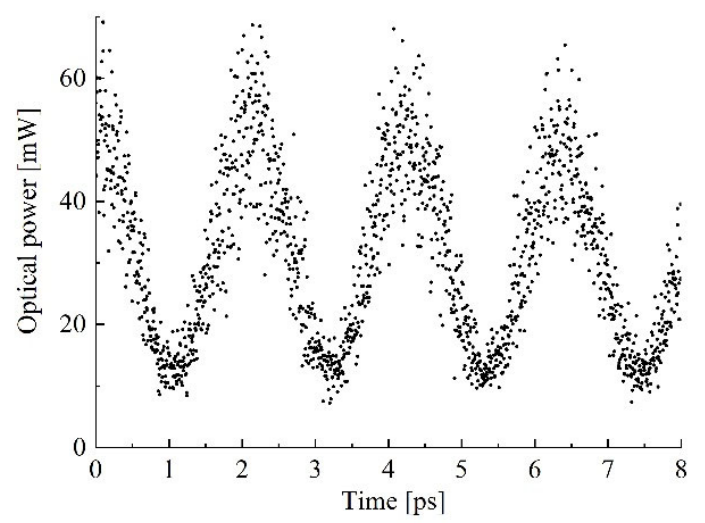

Figure $6470 \mathrm{GHz}$ signal generated by external modulation.

Stable Phase-locked optical spectral components can be generated by using optical external modulators, where the stability is almost same as the electric signal source. Although the frequency response of the modulator is limited up to $100 \mathrm{GHz}$, high-frequency signal generation whose frequency is up to a few hundred $\mathrm{GHz}$ can be generated by using high order sidebands in optical phase modulated signals [37, 38]. The $\mathrm{THz}$ signal generated from the optical sidebands is very stable in frequency domain as well as in time domain. A reciprocating optical modulator (ROM), consisting of a pair of optical filters (input and output filters) and an optical phase modulator, can generate $\mathrm{THz}$ carrier components [37]. Desired optical sideband components would be enhanced by 
the reciprocating modulation process where intermediate sideband components are confined between the filters. Fig. 7 shows a time domain profile of a $470 \mathrm{GHz}$ signal generated by the ROM [39].

\section{ADVANCED ELECTRONICS FOR THZ COMMUNICATIONS}

The state-of-the-art $\mathrm{THz}$ technologies at $300 \mathrm{GHz}$ can provide $100 \mathrm{~Gb} / \mathrm{s}$ high-speed transmission, without losing the spectral efficiency, as discussed in Section II. However, most of the results reported recently based on off-line signal processing to show feasibility of such $\mathrm{THz}$ links, where a real-time transmission should be realized for actual applications.

To offer a real-time $\mathrm{THz}$ transmission, the Horizon 2020 EU-Japan project ThoR ("TeraHertz end-to-end wireless systems supporting ultra high data Rate applications") will develop an over $100 \mathrm{~Gb} / \mathrm{s}$ radio transmission system using frequency band beyond $275 \mathrm{GHz}$ [40].

The ThoR aims to realize high-speed wireless transmission using key enabling $\mathrm{THz}$ technologies. An ultra-broadband, high linearity $\mathrm{THz}$ transmitter at $300 \mathrm{GHz}$ will be demonstrated by using photonic local oscillator and multifunctional $\mathrm{THz}$ integrated semiconductor circuits for upconversion and medium power amplification. $300 \mathrm{GHz}$ radio signals will be generated from V- or E-band IF signals, where $\mathrm{V}$ - or E-band modems will be used for IF-signal generation from real-time baseband data streams. A Traveling-Wave Tube Amplifier (TWTA) will be required for km-range transmission [41]. A $300 \mathrm{GHz}$ receiver will be developed by using multi-functional $\mathrm{THz}$ integrated circuits with high dynamic range, low noise figure and high spectral purity photonic local oscillator generation. The expected output power of the TWTA is $1 \mathrm{~W}$ at $300 \mathrm{GHz}$, where the integrated semiconductor circuit would generate over $10 \mathrm{~mW}$ signals to drive the TWTA. The photonic LO signal can be easily distributed over fibers without losing purity of the signal.

Photonics would be useful for generation and distribution of $\mathrm{THz}$ signals over optical fibers. High-speed semiconductor integrated circuits are required for $\mathrm{THz}$ generation and detection at RF-frontends. TWTAs can amplify the $\mathrm{THz}$ signals up to $1 \mathrm{~W}$. Combination of such various types of components including photonic devices, compound semiconductors and vacuum tubes, are required for demonstration of over $100 \mathrm{~Gb} / \mathrm{s} \mathrm{THz}$ links.

\section{CONCLUSION}

This paper provides research trends on $\mathrm{THz}$ and photonic seamless networks which would be useful for future mobile systems, where a number of BSs and RAUs should be effectively connected. CFSE, an index for contribution to mitigation of spectrum congestion, implies that the state-ofthe-art $\mathrm{THz}$ radio links would provide $100 \mathrm{~Gb} / \mathrm{s}$ high-speed transmission by the use of $300 \mathrm{GHz}$ frequency region, without losing the spectral efficiency.

The Horizon 2020 EU-Japan project ThoR will demonstrate real-time $\mathrm{THz}$ transmission by using high-speed electronics and photonics. The peak in of the CFSE shows the status of the state-of-the art $\mathrm{THz}$ technology and the frontline of the $\mathrm{THz}$ research. The peak frequency can be increased by developing high-power and pure $\mathrm{THz}$ signal generation, with various types of electric and photonic devices.

\section{ACKNOWLEDGMENT}

This paper includes research results from works funded by the MIC/SCOPE \#195003004 and JSPS Grants-in-Aid for Scientific Research Grant Number JP18H01454. The Thor project described in Section V is funded by Horizon 2020, the European Union's Framework Programme for Research and Innovation, under grant agreement No. 814523, and by the Commissioned Research (No. 196) of National Institute of Information and Communications Technology (NICT), Japan. This work was also partially supported by Waseda Research Institute for Science and Engineering

\section{REFERENCES}

[1] 5G PPP Architecture Working Group, "View on 5G architecture".

[2] T. Kawanishi, A. Kanno, H. S.C. Freire, "Wired and Wireless Links to Bridge Networks: Seamlessly Connecting Radio and Optical Technologies for 5G Networks," IEEE Microwave Magazine, 19 (3), 102-111 (2018).

[3] T. Kawanishi, "THz and Photonic Seamless Communications," IEEE/OSA J. Lightwave Technol. 37, 1671-1679 (2019)

[4] P. T. Dat, A. Kanno, T. Umezawa, N. Yamamoto and T. Kawanishi, "Millimeter- and terahertz-wave radio-over-fiber for 5G and beyond", IEEE Photonics Society Summer Topical Meeting Series 2017.

[5] K. Liu, S. Jia, S. Wang, X. pang, W. Li, S. Zheng, H. Chi, X. Jin, X. Zhang and X. Yu, "100 Gbit/s THz Photonic Wireless Transmission in the 350-GHz Band with Extended Reach," IEEE Photon. Technol. Lett., 30, 1064-1067 (2018).

[6] E. Lacombe, C. Belem-Goncalves, C. Luxey, F. Gianesello, C. Durand, D. Gloria and G. Ducournau., "10-Gb/s Indoor THz Communications Using Industrial Si Photonics Technology," IEEE Microwave and Wireless Components Lett.., 28, 362-364 (2018).

[7] A. Stöhr, M. F. Hermelo, M. Steeg, P. T. B. Shih and A. Ng'oma, "Coherent radio-over-fiber $\mathrm{THz}$ communication link for high datarate $59 \mathrm{Gbit} / \mathrm{s}$ 64-QAM-OFDM and real-time HDTV transmission," OFC 2017, Tu3B.

[8] M. Fujishima, "Terahertz wireless communication using $300 \mathrm{GHz}$ CMOS transmitter", IEEE ICSICT 2016.

[9] P. Latzel, F. Pavanello, S. Bretin, M. Billet, E. Peytavit, C. Coinon, X. Wallart, JF Lampin, M. Zaknoune, G. Ducournau1 "High efficiency UTC Photodiode for High Spectral Efficiency THz links", IEEE IRMMW-THz 2017.

[10] X. Yu, Shi Jia, X. Pang, T. Morioka and L. K. Oxenloewe, "Beyond 100 Gbit/s Wireless Connectivity Enabled by THz Photonics", ICTON 2017, WeA2.3.

[11] S. Jia, X. Yu, H. Hu, J. Yu, T. Morioka, P. U. Jepsen, and L. K. Oxenløwe, "80Gbits/s 16-QAM Multicarrier $\mathrm{THz}$ Wireless Communication Link in the $400 \mathrm{GHz}$ Band", ECOC 2016.

[12] X. Li, J. Yu, J. Zhang, Z. Dong, F. Li and N. Chi, Opt. Express 21, 187894-187899 (2013).

[13] A. Hirata, T. Kosugi, H. Takahashi, J. Takeuchi, H. Togo, M. Yaita, N. Kukutsu, K. Aihara, K. Murata, Y. Sato, T. Nagatsuma and Y. Kado, IEEE Trans. Microw. Theory Tech., 60, 881-895 (2012).

[14] M. Fujishima, S. Amakawa, K. Takano, K. Katayama and T. Yoshida, "Terahertz CMOS Design for Low-Power and High-Speed Wireless Communication,” IEICE Trans. Electron. E98-C 1091-1104 (2015).

[15] H. Shams, M. J. Fice, K. Balakier, C. C. Renaud, F. Dijk, and A. J. Seeds, "Photonic generation for multichannel $\mathrm{THz}$ wireless communication," Opt. Express 22, 23465-23472 (2014)

[16] S. Koenig, D. Lopez-Diaz, J. Antes, F. Boes, R. Henneberger, A. Leuther, A. Tessmann, R. Schmogrow, D. Hillerkuss, R. Palmer, T. Zwick, C. Koos, W. Freude, O. Ambacher, J. Leuthold and I. Kallfass, "Wireless sub-THz communication system with high data rate," Nature Photon. 7, 977-981 (2013).

[17] I. Kallfass, F. Boes, T. Messinger, J. Antes, A. Inam, U. Lewark, A. Tessmann, R. Henneberger, "64 Gbit/s Transmission over $850 \mathrm{~m}$ Fixed Wireless Link at $240 \mathrm{GHz}$ Carrier Frequency,” J. Infrared Milli. Terahz Waves 36, 221-233 (2015).

[18] I. Kallfass, I. Dan, S. Rey, P. Harati, J. Antes, A. Tessmann, S. Wagner, M. Kuri, R. Weber, H. Massler, A. Leuther, T. Merkle and 
T. Kürner, "Towards MMIC-Based $300 \mathrm{GHz}$ Indoor Wireless Communication Systems," IEICE Trans. Electron. E98-C, 1081-1088 (2015).

[19] A. Kanno, T. Kuri, I. Morohashi, I. Hosako, T. Kawanishi, Y. Yoshida and K.-I. Kitayama, "Coherent Terahertz Wireless Signal Transmission Using Advanced Optical Fiber Communication Technology," J. Infrared Milli. Terahz Waves 36, 180-197 (2015).

[20] T. Nagatsuma, S. Horiguchi, Y. Minamikata, Y. Yoshimizu, S. Hisatake, S. Kuwano, N. Yoshimoto, J. Terada, and H. Takahash, "Terahertz wireless communications based on photonics technologies," Opt. Express 21, 477-487 (2013).

[21] C. Wang, B. Lu, C. Lin, Q. Chen, L. Miao, X. Deng and J. Zhang, "0.34- THz wireless link based on high-order modulation for future wireless local area network applications," IEEE Trans. Terahz Sci. Technol. 4, 75-85 (2014).

[22] G. Ducournau, K. Engenhardt, P. Szriftgiser, D. Bacquet, M. Zaknoune, R. Kassi, E. Lecomte and J.-F. Lampinet, “32 Gbit/s QPSK transmission at $385 \mathrm{GHz}$ using coherent fibre-optic technologies and THz double heterodyne detection," Electron. Lett. 12, 915-917 (2015)

[23] G. Ducournau, P. Szriftgiser, A. Beck, D. Bacquet, F. Pavanello, E. Peytavit, M. Zaknoune, T. Akalin, and J.-F. Lampinet, "UltrawideBandwidth Single-Channel $0.4-\mathrm{THz}$ Wireless Link Combining Broadband Quasi-Optic Photomixer and Coherent Detection," IEEE Trans. Terahz Sci. Technol. 4, 328-337 (2014).

[24] T. Nagatsuma and G. Carpintero, "Recent Progress and Future Prospect of Photonics-Enabled Terahertz Communication Research," IEICE Trans. Electron. E98-C, 1060-1070 (2015).

[25] X. Yu, R. Asif, M. Piels, D. Zibar, M. Galili, T. Morioka, P. U. Jepsen and L. K. Oxenloewe, "60 Gbit/s $400 \mathrm{GHz}$ wireless transmission," IEEE International Conference on Photonics in Switching 2015.

[26] L. Moeller, J. Federici and K. Su et al., "2.5Gbit/s duobinary signalling with narrow bandwidth 0.625 terahertz source," Electron. Lett. 47, 856-858 (2011).

[27] H. Shams, T. Shao, M. J. Fice, P. M. Anandarajah, C. C. Renaud, F. Van Dijk, L. P. Barry, and A. J. Seeds, "100 Gb/s multicarrier THz wireless transmission system with high frequency stability based on a gain-switched laser comb source," IEEE Photonics J. 7(3), 7902011 (2015)

[28] T. Nagatsuma, K. Kato an J. Hesler "Enabling Technologies for Realtime 50-Gbit/s Wireless Transmission at $300 \mathrm{GHz}$,'NANOCOM' 15 , article no. 10 .

[29] N. Oshima, K. Hashimoto, S. Suzuki and M. Asada, "Terahertz Wireless Data Transmission with Frequency and Polarization Division Multiplexing Using Resonant-Tunneling-Diode Oscillators," IEEE Trans. Terahz Sci. Technol. 7, 593-598 (2017).

[30] K. Katayama, K. Takano, S. Amakawa, S. Hara, T. Yoshida and M. Fujishima, "CMOS 300-GHz 64-QAM transmitter," 2016 IEEE MTT-S International Microwave Symposium (IMS), San Francisco, CA, 2016, pp. 1-4.

[31] N. Sarmah J. Grzyb, K. Statnikov, S. Malz, P. R. Vazquez, W. Föerster, B. Heinemann, and U. R. Pfeifferet al., "A Fully Integrated 240-GHz Direct-Conversion Quadrature Transmitter and Receiver Chipset in SiGe Technology," IEEE Trans. Microw. Theory Tech., 64, 562-574 (2016).

[32] K. Wang, X. Li, M. Kong, P. Gou, W. Zhou and J. Yu, "Probabilistically Shaped 16QAM Signal Transmission in a Photonics-aided Wireless Terahertz-Wave System," 2018 Optical Fiber Communications Conference and Exposition (OFC), San Diego, CA, 2018, pp. 1-3.

[33] S. Jia, X. Pang, O. Ozolins, X. Yu, H. Hu, J. Yu, P. Guan, F. D. Ros, S. Popov, G. Jacobsen, M. Galili, T. Morioka, D. Zibar, and L. K. Oxenløwe, "0.4 THz Photonic-Wireless Link With 106 Gb/s Single Channel Bitrate," IEEE/OSA J. Lightwave Technol. 36, 610-616 (2018)

[34] N. Saito, T. Tsukizawa, N. Shirakata, T. Morita, K. Tanaka, J. Sato, Y. Morishita, M. Kanemaru, R. Kitamura, T. Shima, T. Nakatani, K. Miyanaga, T. Urushihara, H. Yoshikawa, T. Sakamoto, H. Motozuka, Y. Shirakawa, N. Yosoku, A. Yamamoto, R. Shiozaki, and K. Takinami, Member, "A Fully Integrated 60-GHz CMOS Transceiver Chipset Based on WiGig/IEEE 802.11ad With Built-In Self Calibration for Mobile Usage," IEEE J. Solid-State Circuits, 48, 31463159 (2013)

[35] A. Siligaris, O. Richard, B. Martineau, C. Mounet, F. Chaix, R. Ferragut, C. Dehos, J. Lanteri, L. Dussopt, S. D. Yamamoto, R. Pilard, P. Busson, A. Cathelin, D. Belot and P. Vincent, "A 65-nm CMOS Fully Integrated Transceiver Module for $60-\mathrm{GHz}$ Wireless HD Applications," IEEE J. Solid-State Circuits, 46, 3005-3017 (2011)
[36] APT Report on Wired and Wireless Seamless Connections using Millimeter-Wave Radio over Fiber Technology for Resilient Access Networks, APT/ASTAP/REPT-11.

[37] T. Kawanishi, T. Sakamoto and M. Izutsu, "High-Speed Control of Lightwave Amplitude, Phase, and Frequency by Use of Electrooptic Effect," IEEE J. Sel. Topics in Quantum Electron., 13, 79-91 (2007).

[38] H. Kiuchi, T. Kawanishi, M. Yamada, T. Sakamoto, M. Tsuchiya, J. Amagai, and M. Izutsu, "High Extinction Ratio Mach-Zehnder Modulator Applied to a Highly Stable Optical Signal Generator," IEEE Trans. Microwave Theory Tech., 55, 1964-1972 (2007)

[39] T. Kawanishi, T. Sakamoto and M. Izutsu, "470GHz Optical Clock Signal Generation Using Reciprocating Optical Modulation,” 2006 European Conference on Optical Communications (ECOC)

[40] www.ThoRProject.eu

[41] K. Tsutaki, Y. Neo, H. Miura, N. Masuda, M. Yoshida, "Design of a $300 \mathrm{GHz}$ Band TWT with a Folded Waveguide Fabricated by Microelectromechanical Systems," Journal of Infrared, Millimeter, and Terahertz Waves, 37, 1166-1172 (2016) 